\title{
Quantitative Electroencephalography (Q-EEG) map of parietal and frontal lobes out of brain waves recording during dental hypnosis practice
}

\author{
Gilang Yubiliana ${ }^{1 *}$, Raka Putra ${ }^{2}$, Andri Abdurrochman ${ }^{3}$ \\ ${ }^{1 *}$ Department of Public Health, Faculty of Dentistry, Universitas Padjadjaran, Indonesia \\ ${ }^{2}$ Faculty of Dentistry, Universitas Padjadjaran, Indonesia \\ ${ }^{3}$ Department of Physics, Faculty of Mathematics and Natural Sciences, Universitas Padjadjaran, \\ Indonesia
}

\begin{abstract}
Introduction: A patient with fear and anxiety is a common case to deal with for a dentist, therefore, dental hypnosis has been widely used to ease this situation. In a hypnotized state, the human brain may easily accept any suggestion. This is projected in the brain waves. Electroencephalograph (EEG) is a brain wave recording device, reflecting several states of consciousness. Beta for conscious, alpha and theta for subconscious, and delta for sleep. Dental hypnosis puts down beta waves to alpha or theta. Quantitative Electroencephalography (Q-EEG) or brain mapping is a comprehensive analysis of (Electroencephalography, EEG) in a colored topographic map, reflecting the brain's electrical activity. The objective of this article was reporting the parietal and frontal lobes activity during dental hypnosis based on the Q-EEG mapping. Methods: The research applied a quantitative research method using observatory study. The sample was taken with an accidental sampling method, with inclusion criteria, patients with dental anxiety and exclusion criteria was patients with special need and high level of dental anxiety. Data of the EEG records was taken in January-March 2018, and processed after in Pramita laboratorium Bandung. Results: Parietal lobe affected more during the inducement than temporal lobe. During dental hypnosis, the hypnotic markers (theta and alpha states) observed from the EEG were found to be more reactive. Conclusion: Dental hypnosis effects can be observed easily using Quantitative Electroencephalography method. Dental hypnosis affects brainwaves and brain mapping which indicate relaxations of brain waves especially on parietal lobes.
\end{abstract}

Keywords: Quantitative electroencephalography; brain mapping; brainwave; dental hypnosis

p-ISSN: 1979-0201; e-ISSN: 2549-6212; Available from: http://jurnal.unpad.ac.id/pjd/article/view/33382

DOI: $10.24198 /$ pjd.vol33no2.33382

Submission: Apr 16, 2019; Accepted: Nov 30, 2021; Published online: Nov 30, 2021

*Corresponding author: Gilang Yubiliana, Department of Dental Public Health, Faculty of Dentistry Universitas Padjadjaran, Indonesia. Jalan Sekeloa Selatan I, Bandung, West Java, 40132, Indonesia. Phone: +62 815-7007-021;

Email: gilang.yubiliana@fkg.unpad.ac.id 


\section{INTRODUCTION}

There are so many responses shown by patients who visit dental clinics for treatment. A common struggle often experienced by dentists is patients who come with fear and anxiety. Hypnosis is known to help this struggle and has been widely used to treat anxiety during dental treatment. $.^{12},{ }^{12}$ Dental hypnosis is a sleep-like condition or a subconscious state of mind, a form of psychotherapy that aids in relaxation, reducing anxiety and fear. In a hypnotized condition, the human brain can easily accept suggestions and input, able to effectively change a patient's physiological and neurological functions, thus increasing the effectiveness of dental treatment. In Physics, this condition is closely related to the brain waves. ${ }^{2,13}$

Living human brain produces fluctuating electrical waves, known as brain waves. Human brain produces various kinds of brain waves simultaneously. Based on the magnitude of the frequency, the brain produces beta, alpha, theta, and delta waves. The most dominant brain wave indicates the brain or mental activity in that moment. Electroencephalography (EEG) is a brain wave measuring device, a tool able to record electrical activity in the brain using electrodes placed on the scalp. EEG checks the brain waves, vibrations, signals, and frequencies, then grouped them into several states of consciousness. $3,4,14$ Beta waves reflect consciousness or normal state, alpha and theta waves reflect subconsciousness or a hypnotized state, and delta waves reflect a normal sleep state. Hypnosis brings the patient's awareness from conscious to subconscious, from beta waves to alpha or theta waves, but not to reach delta waves. ${ }^{2,16}$

Brain mapping, technically known as Quantitative Electroencephalography (Q-EEG), is a comprehensive analysis of the wave's frequencies recorded by the EEG.5,15 Obtained data will be shown as a colored topographic map, reflecting the brain's electrical activity. ${ }^{6,15,16}$ This article continues previous research carried in 2016 regarding body's physiological responses during dental hypnosis using electroencephalographic records. Using the brain mapping method, this article aims to see the brain's physiological responses mapping of the frontal and temporal lobes based on the recorded brain waves to find out the brain's electrical activity during dental hypnosis. The objective of this article was reporting the parietal and frontal lobes activity during dental hypnosis based on the Q-EEG mapping.

\section{METHODS}

The research applied a quantitative research method using observatory study. The sample was taken with an accidental sampling method, with inclusion criteria, patients with dental anxiety and exclusion criteria was patients with special need and high level of dental anxiety. Data of the EEG records was taken in January-March 2018, and processed after in Pramita laboratorium Bandung. Research samples consist of analysis results from EEG recordings, the increasing or decreasing ratios of brain wave frequencies recorded in the form of high, medium, and low frequencies, as well as the brain wave types, namely beta, alpha, theta, and delta.

Research preparation stage includes collecting data from previous studies, EEG recordings and completion in research materials and tools such as laptop or computer and the data. Research data processing procedures includes analyzing EEG data records sequentially with a minimum time of 10 seconds, then classifying it based on its frequency and their brain wave sizes such as beta, alpha, theta, and delta. Later, it will be classified into high, medium, and low frequencies. The results will be presented in the form of an RGB color spectrum, such as blue for low frequencies, green for medium frequencies, and red for high frequencies, which is then converted into RGB hex to determine each electrode's color. Brain mapping based on the color then made on each electrode sequentially with a minimum of 10 seconds. The final result is an RGB colored topographic map showing brain electrical activity on each electrode.

Subjects in this research consist of 2 men and 2 women with an 18-24 years old age range. EEG signals recorded using the NicoletOne EEG instrument with 10-20 electrode channel installation systems, with a total of 21 electrodes. After electrodes were placed, hypnosis and scaling were carried out on each subject, then EEG signal recording results were obtained from 
Nicolet Datafile Software. Recorded EEG signal data then converted into ASCII data. These data are then adjusted according to the time of each sentence stimulation and processed using the PSD methodology implemented through the Matlab program.

The percentage of the power spectrum area of each frequency is then calculated and divided into low (delta), medium (alpha and theta), and high (combining beta and gamma) frequencies. These data then converted into RGB (red, green blue) colors by changing the percentage data of each frequency into RGB Hex code and the dominant color was obtained for each installed electrode channel. Blue for low frequencies, green for medium frequencies, and red for high frequencies. The dominant color obtained then visualized into brain mapping by giving colors according to the RGB hex code on each electrode channel. Brain mapping images in the form of a topographic map then analyzed and correlated with the physiological responses of the brain according to the location of the affected electrode channels, and color changes in each electrode channel were also analyzed according to the time segmentation of the hypnosis process.

\section{RESULTS}

Subject's hypnotized state was marked by the dominance of medium frequency, or theta and alpha waves on the parietal lobes of the brain . Subject 1 started to get hypnotized by Sentence 8 , marked by $\mathrm{P} 4$ as the most dominant, in line with Evanti in 2017 and Gunawan in 2017., 7 On Subject 1 , the brain part significantly induced was $\mathrm{P} 3$ as much as 8 times (50\%) and P4 as much as 7 times (43.7\%).

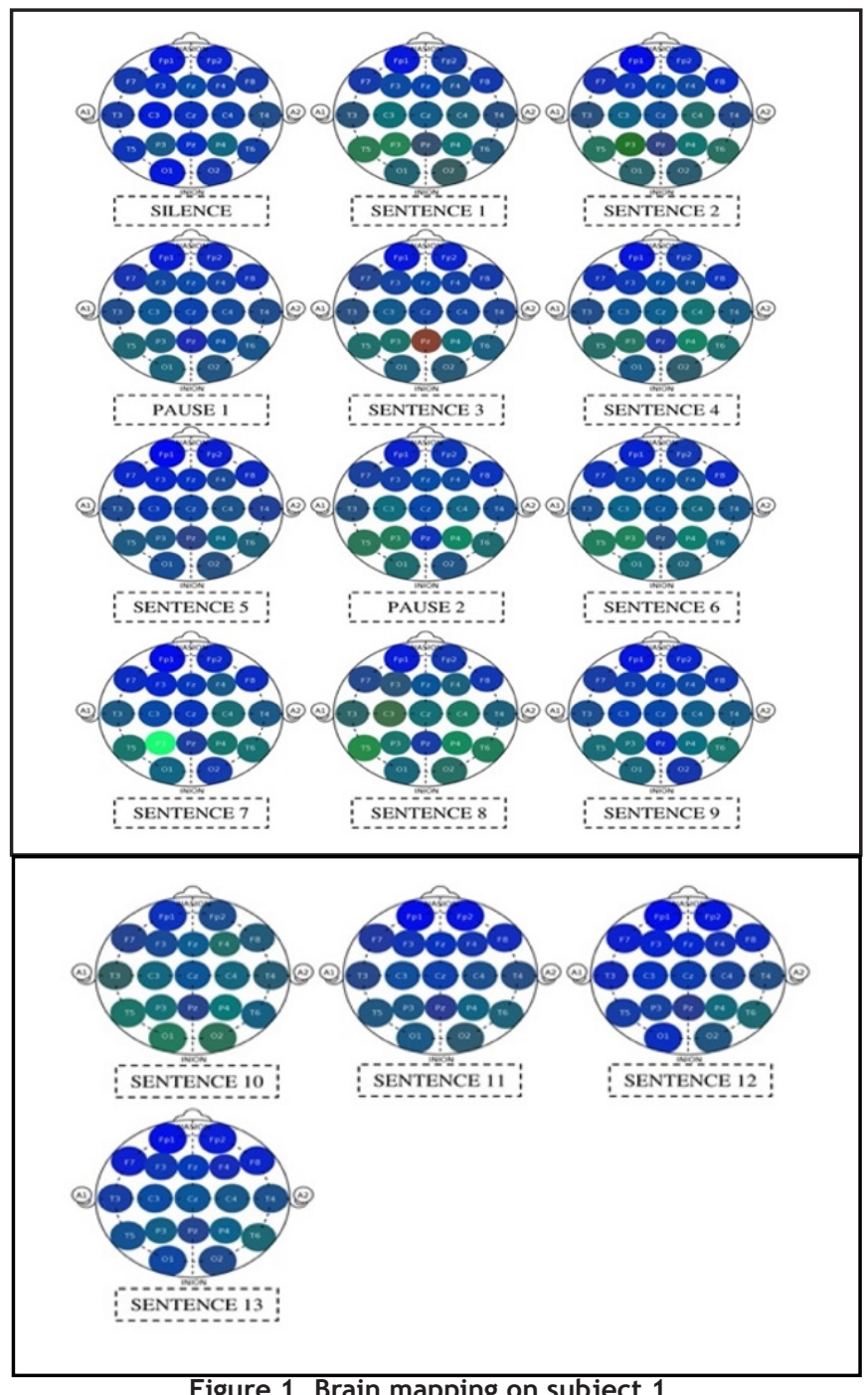

Figure 1. Brain mapping on subject 1 
Subject 2 started to get hypnotized by Sentence 19 , marked by P3 as the most dominant, but this is not in line with Evanti (2017) and Gunawan (2017) which stated that Subject 2 started to get hypnotized by Sentence 23.,8 On Subject 2, the brain part significantly induced was $\mathrm{P} 3$ as much as 30 times (88.2\%), 01 as much as 13 times, and P4 as much as 16 times.
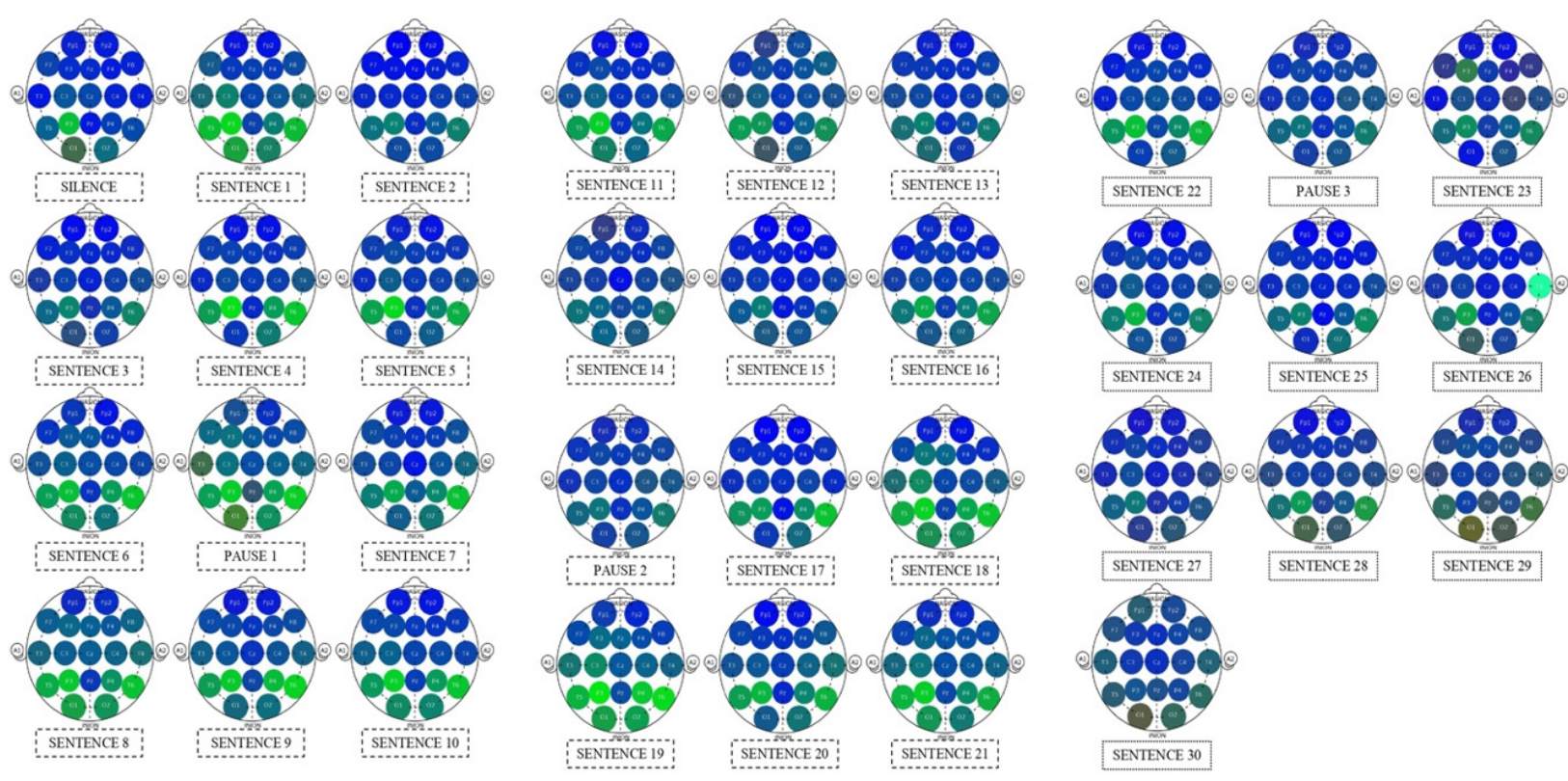

Figure 2. Brain Mapping on Subject 2

Subject 3 started to get hypnotized by Sentence 4, marked by $\mathrm{P} 3$ as the most dominant, in line with Evanti (2017 and Gunawan (2017). ${ }^{7,8}$ On Subject
3 , the brain part significantly induced was P3 as much as 22 times (84.6\%), 01 as much as 24 times (92.3\%), and T5 as much as 22 times (84.6\%).

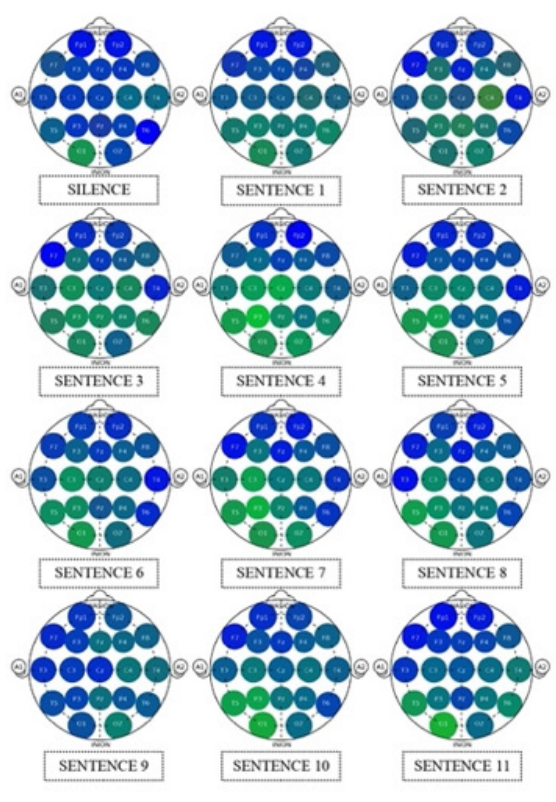

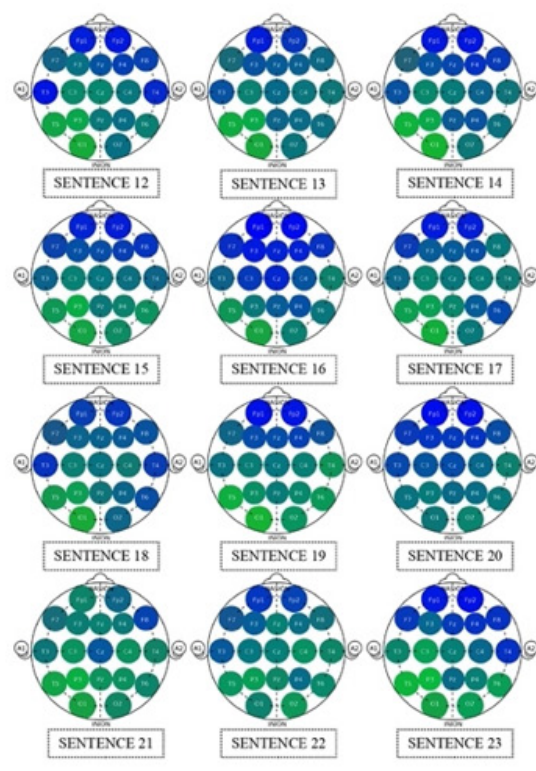

Figure 3. Brain Mapping on Subject 3
Subject 4 started to get hypnotized by Sentence 14 , marked by $\mathrm{P} 3$ as the most dominant, in line with Evanti (2017) and Gunawan (2017). ${ }^{7,8}$ On Subject 4, the brain part significantly induced was
P4 as much as 28 times (90.3\%), P3 as much as 26 times (83.8\%), $\mathrm{O} 2$ as much as 30 times (96.7\%), F4 and F8 as much as 29 times (93.5\%). 


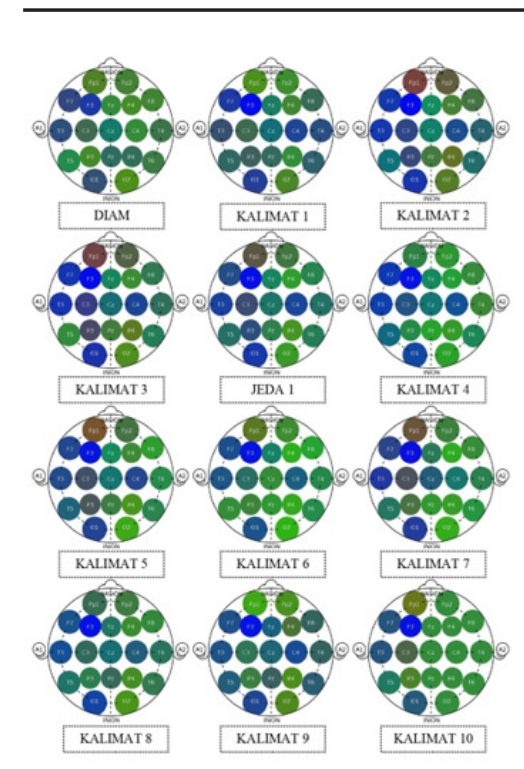

\section{DISCUSSION}

Based on the results, it was found that Subject 1 started to get hypnotized by Sentence 8 , marked by $\mathrm{P} 4$ as the most dominant and there was no visible marking on the frontal area. Subject 2 started to get hypnotized by Sentence 19 , marked by $\mathrm{P} 3$ as the most dominant and on the frontal part was marked by $F 3$ on Sentence 23 . Subject 3 started to get hypnotized by Sentence 4 , marked by $\mathrm{P} 3$ as the most dominant and on the frontal part was marked by Fp1 on Sentence 21. Subject 4 started to get hypnotized by Sentence 14, marked by $\mathrm{P} 3$ as the most dominant and on the frontal part was marked by F4 on Sentence 14 .

The most induced parietal electrode channel during dental hypnosis on Subject 1 was $\mathrm{P} 3$ as much as 8 times $(50 \%)$, on Subject 2 was $\mathrm{P} 3$ as much as 30 times $(88.2 \%)$, on Subject 3 was $P 3$ as much as 22 times (84.6\%), and for Subject 4 was P4 as much as 28 times (90.3\%). In this study, three subjects showed that P3 was most induced during dental hypnosis and only one subject showed P4. Parietal lobe itself functions as the central area of sensory awareness. The posterior parietal cortex plays an important role in the integration of visuomotor, spatial perception, spatial orientation, aspects of attention, and visual motor analysis. ${ }^{9}$ This is in line with Tebecis et al (1990), which stated that patients with high susceptibility produces more theta activity in the parietal part of both hemispheres, during waking and hypnosis, in both eyes open and closed. ${ }^{10}$ This is also in line with Fingelkurts et al (2007) which showed a fairly large distribution of theta and alpha brain waves on the parietal area. ${ }^{10}$ Whereas in the frontal area, on Subject $1 \mathrm{~F} 4$ appeared once (6.25\%), on Subject 2 F3 appeared once (2.9\%), on Subject $3 \mathrm{~F} 3$ appeared 3 times (11.5\%), and on Subject 4 F4 appeared 29 times (93.5\%).

The affected hemisphere of three subjects were left parietal and one subject was right parietal. According to Cappelletti (2010), the left and right parietal play different roles in numerical processing, the left parietal cortex is more involved in precise and language-dependent number processing, while the right cortex is more involved in processing approximate numbers. Left parietal activation reflects various processes associated with the extraction and comparison of gathered information, whereas right parietal number activation is more involved in the conceptual processing of numbers rather than object names. ${ }^{11}$

As a whole, this study showed appearance on hypnotic markers were more significant on the parietal area, characterized by the appearance of dominant medium frequency, and the parietal area was also more induced during dental hypnosis. As for the frontal area, there was no sign of hypnotic marker, not in line with previous studies which stated that the frontal area is also induced marked by the increasing dynamics of the medium frequency. ${ }^{7,8}$ But similar study needs to be carried 
out using other brain mapping methods and a study which differentiates between hypnotized patients and non-hypnotized patients.

\section{CONCLUSION}

Dental hypnosis effects can be observed easily using Quantitative Electroencephalography method. Dental hypnosis affects brainwaves and brain mapping which indicate relaxations of brain waves especially on parietal lobes.

\section{ACKNOWLEDGEMENTS}

This study was funded by RKDU phase 1 Universitas Padjadjaran, in 2019.

\section{REFERENCES}

1. Destiarlisa AM, Yubiliana $G$, Abdurrochman A, Darmayanti N. Intonation pattern of directive speech acts based on sound acoustic parameters in the practice of dental hypnosis. Padjadjaran J Dent Res. 2020; 4(1): 1-8. DOI: 10.24198/pjdrs.v4i1.21989

2. Yubiliana G. The Effectiveness of Dental Hypnosis - Komunika Hipnodontik to Salivary Cortisol Hormone Levels as Dental Anxiety Biomarker and Its Correlation with Quality of Life. [Thesis]. Padjadjaran University; 2016.

3. Hillmer K, Kappesser J, Hermann C. Pain modulation by your partner: An experimental investigation from a socialaffective perspective. PLoS One. 2021 Jul 22;16(7): e0254069. DOI: 10.1371/journal. pone. 0254069.

4. Xu Q, Ye C, Gu S, Hu Z, Lei Y, Li X, Huang L, Liu Q. Negative and Positive Bias for Emotional Faces: Evidence from the Attention and Working Memory Paradigms. Neural Plast. 2021; 2021: 8851066. DOI: 10.1155/2021/8851066.

5. Campbell BA, Machado AG, Baker KB. Electrophysiologic mapping for deep brain stimulation for movement disorders. Handb Clin Neurol. 2019; 160: 345-55. DOI: $10.1016 /$ B978-0-444-64032-1.00022-9.

6. Popa LL, Dragos H, Pantelemon C, Rosu OV, Strilciuc S. The Role of Quantitative EEG in the Diagnosis of Neuropsychiatric Disorders. J Med Life. 2020;13(1).
7. Kusmayanti S, Yubiliana G, Abdurrochman A, Ramdani ML, Fauzan NH. Effectiveness test of dental hypnosis monitoring device. Padjadjaran J Dent. 2021; 33(1): 26-31. DOI: 10.24198/pjd.vol33no1.22383

8. Appukuttan DP. Strategies to manage patients with dental anxiety and dental phobia: literature review. Clin Cosmet Investig Dent. 2016; 8: 35-50. DOI: 10.2147/CCIDE.S63626. eCollection 2016

9. Olivers CNL, Roelfsema PR. Attention for Action in Visual Working Memory Author Links Open Overlay Panel. Cortex. 2020; 131(Special Issue).179-194. DOI: 10.1016/j. cortex.2020.07.011

10. Keshmiri S, Alimardani $M$, Shiomi $M$, Sumioka $\mathrm{H}$, Ishiguro $\mathrm{H}$, Hiraki $\mathrm{K}$. Higher hypnotic suggestibility is associated with the lower EEG signal variability in theta, alpha, and beta frequency bands. PLOS ONE. 2020; 15(4): e0230853. DOI: 10.1371/journal. pone. 0230853

11. Wild HM, Heckemann RA, Studholme C, Hammers A. Gyri of the human parietal lobe: Volumes, spatial extents, automatic labelling, and probabilistic atlases. PLOS ONE. 2017; 12(8): e0180866. DOI: 10.1371/journal. pone. 0180866

12. Ahlskog G. Clinical Hypnosis Today. Psychoanal Rev. 2018; 105(4): 425-37. DOI: $10.1521 /$ prev.2018.105.4.425.

13. Chertok L. Hypnosis and suggestion in a century of psychotherapy: an epistemological assessment. JAmAcad Psychoanal. 1984; 12(2): 211-32. DOI: 10.1521/jaap.1.1984.12.2.211 .

14. (Lynn SJ, Kirsch I, Barabasz A, Cardeña E, Patterson D. Hypnosis as an empirically supported clinical intervention: the state of the evidence and a look to the future. Int J Clin Exp Hypn. 2000; 48(2): 23959. DOI: 10.1080/00207140008410050. Biasiucci A, Franceschiello B, Murray MM. Electroencephalography. Curr Biol. 2019; 29(3): 80-5. DOI: 10.1016/j.cub.2018.11.052.

15. Omejc N, Rojc B, Battaglini PP, Marusic U. Review of the therapeutic neurofeedback method using electroencephalography: EEG Neurofeedback. Bosn J Basic Med Sci. 2019; 19(3): 213-20. DOI: 10.17305/ bjbms.2018.3785. 